\title{
Experimental and numerical modelling of mass transfer in a refining ladle
}

\author{
Nelson Joubert ${ }^{1, *}$, Pascal Gardin ${ }^{2}$, Stéphane Popinet ${ }^{1}$, and Stéphane Zaleski ${ }^{1,3}$ \\ ${ }^{1}$ Sorbonne Université and CNRS, UMR 7190, Institut Jean Le Rond d'Alembert, BP 162, 4, Place Jussieu, 75252 Paris \\ Cedex 05, France \\ 2 ArcelorMittal Maizières Research, BP 30320, Voie Romaine, 57283 Maizières-lès-Metz Cedex, France \\ ${ }^{3}$ Institut Universitaire de France, Paris, France
}

Received: 2 July 2021 / Accepted: 8 November 2021

\begin{abstract}
Mass transfer between liquid steel and slag is an important physical phenomenon during secondary metallurgy for prediction of the chemical reaction rate and adjustment of liquid steel composition. We study this phenomenon at ambient temperature with a water experiment and perform Direct Numerical Simulations, aiming to reproduce an argon-gas bottom-blown ladle. First, we measure the evolution of the time-averaged open-eye area as a function of the air flow rate. Both simulation and experiment agree relatively well and are close to other water experiments in the literature. Secondly, the mass transfer of thymol between water and oil is investigated. The experimental results show that two mass transfer regimes can be observed. The regime change coincides with atomization of the oil layer resulting in the continuous formation of oil droplets in the water whenever the air flow rate rises above a critical value. The numerical results for the mass-transfer rate or Sherwood number are obtained at small Schmidt numbers and are then extrapolated to the experimental Schmidt number of 1480. A good agreement with experiment is observed although with large error bars. The Sherwood numbers at the two largest simulated flow rates show a steep increase.
\end{abstract}

Keywords: mass transfer / computational fluid dynamics / volume-of-fluid / large Schmidt numbers / multiphase flow

\section{Introduction}

In numerous natural and industrial processes, chemical reaction is strongly coupled with a fluid flow. In the steel industry, during the secondary metallurgy step, the adjustment of liquid steel composition at high temperature in a ladle involves several chemical reactions between steel and slag [1]. Chemical reactions can be broken down into three stages: transport by convection of reactive species within the liquid phase toward the interface, transport by molecular diffusion through the concentration boundary layer and finally chemical reaction at the interface. The global kinetics is governed by the kinetics of the slowest stage. In the case of a chemical reaction between liquid steel and slag, it is generally accepted that chemical reactions at the interface are very fast. Then, the steps governing the global kinetics are the convective and diffusive transport or mass transfer of reactive species. In order to better adjust the steel composition, it is important to characterize the mass transfer of reactive species between the liquid steel

\footnotetext{
* e-mail: nelson.joubert@sorbonne-universite.fr
}

and slag phase. Here, for example, the process to be studied is the desulfurization of liquid steel by chemical reaction with slag under argon gas stirring. Previous studies attempted to model experimentally the process either with ambient temperature ladle [2-4] or high-temperature ladle $[3,5,6]$. Other studies try to model the process numerically [7-9]. The results showed some change in the mass transfer regime between the two phases when the gas flow rate reaches a particular value. In the literature, it is generally acknowledged that the observed mass transfer regime change is due to the high deformation of the slag layer leading ultimately to its fragmentation into slag droplets in the liquid steel [10-13]. Slag droplets formation would increase the exchange area between the two phases. In order to verify this assumption and the global behavior of the mass transfer, we developed simultaneously an experimental water ladle of the process and its numerical model. Considering constant temperature and fluids properties in the ladle, the gas flow rate is the control parameter of the process. Then we will vary the gas flow rate to measure its influence on the mass transfer. In this study, we start by giving details on the experimental and numerical procedure. Then the result section is divided in 
two parts. The first one is a brief hydrodynamic characterization of the water experiment and, the second one concern the mass transfer characterization.

\section{Model description}

The main characteristics of the flow consist of the interaction of a gas bubble plume with a slag layer at the free surface. Given the numbers of parameters of the physical model, many dimensionless number quantities can be established. As a result, the choice of similitude to follow is not trivial and depends on the phenomena of interest. In this study, we are interested in the characterization of the phenomenon of mass transfer between liquid steel and slag. Nevertheless, this mass transfer depends strongly on the fluid flow characteristics.

\subsection{Experimental model}

If we assume that within the gas flow rate range considered in the industrial case, we are in presence of a bubble plume $[14,15]$, the vertical rising velocity due to the bubble plume in the liquid steel can be approximated by a characteristic velocity scale given by

$$
U=\left(g^{2} Q\right)^{1 / 5}
$$

Now, considering the expression of (1) we can rewrite the Froude number

$$
F r=\frac{U}{\sqrt{g h_{m}}}
$$

as a function of the gas flow rate to obtain the expression of the Froude number $N$

$$
N=\left(\frac{Q}{g^{1 / 2} h_{m}^{5 / 2}}\right)^{1 / 5}
$$

To determine the corresponding gas flow rate between a 180-ton industrial ladle and our experiment a Froude number similarity can be used in which the model experiment and the industrial ladle have the same Froude number.

$$
Q_{i l}=\left(h_{m, i l} / h_{m, r l}\right)^{\frac{5}{2}} Q_{r l}
$$

with $h_{m}$ and $h_{s}$ respectively the height of the steel and the slag equivalent phase. $i l$ and $r l$ are respectively the indices for the industrial and reduced ladle and $Q$ is the gas flow rate considered at the operating temperature and pressure at the injector height. In this study, we use a cubic ladle filled of water with an oil layer at the top. Both oil and water are at ambient temperature. Figure 1 displays a sketch of the experimental setup of the water ladle and the main geometric parameters of the ladle can be found in Table 1. It is a square-section transparent ladle made with acrylic glass, with a single bottom centered circular air

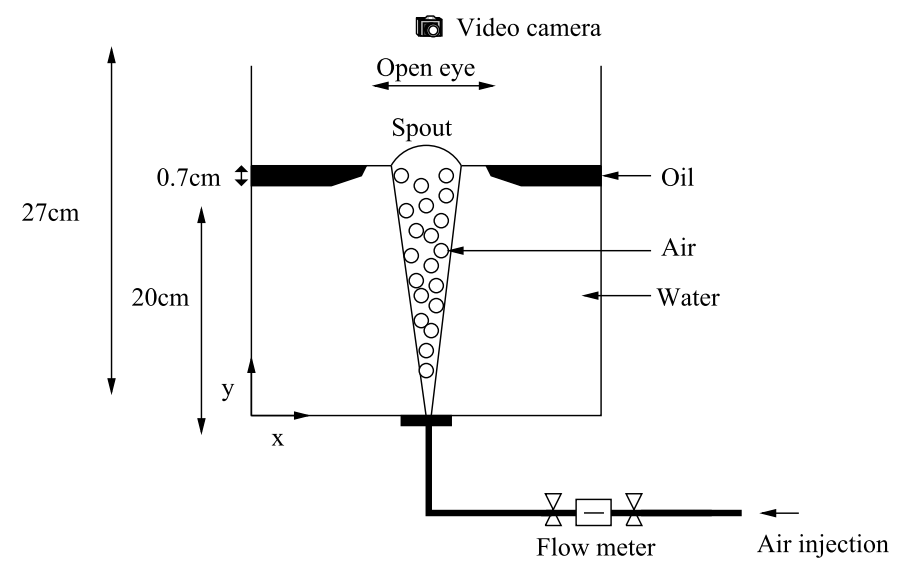

Fig. 1. Schematic of the experimental setup of the water ladle.

injection hole. Air flow is injected from a compressed air network and can be varied through an automate controlling the valve opening. In the following, we denote the water, oil, and air phase with the respective indices $w$, $o$ and $a$.

As in the industrial process, it is a three-phase experiment where the air phase represents the argon gas, water represents the liquid steel, and the oil mixture represents the liquid slag. The fluids choice follows the one used in [2], the oil phase is a 50-50\% in volume mixture of cottonseed oil and paraffin oil. All the fluids physical parameters can be found in Table 2. Following [2], to reproduce the behavior of the Sulphur dissolved in liquid steel we have chosen thymol $\left(\mathrm{C}_{10} \mathrm{H}_{14} \mathrm{O}\right)$ dissolved in water as chemical tracer in our experiment. In the experiment thymol concentration in water is measured every 10 minutes during 3 hours by analyzing water samples with a refractometer previously calibrated. Measurements can be done with two size of injection diameters $d_{i n j}=2.35 \mathrm{~mm}$ and $d_{i n j}=7.9 \mathrm{~mm}$, with $14.4 \mathrm{l} \mathrm{of}$ water with a thymol concentration of $0.9 \mathrm{~g} / \mathrm{l}$ and 0.4861 of oil mixture atop of it.

We detail here the general solution of the evolution of the concentration of thymol in the water. The mass transfer equation of thymol in each phase gives

$$
\begin{gathered}
\frac{d C_{w}}{\mathrm{~d} t}=-\frac{K_{w} A}{V_{w}}\left(C_{w}-C^{\prime}{ }_{w}\right)=J<0 \\
\frac{d C_{o}}{\mathrm{~d} t}=-\frac{K_{o} A}{V_{o}}\left(C_{o}-C^{\prime}{ }_{o}\right)=-J>0
\end{gathered}
$$

where $C_{w}(g / l)$ and $C_{o}(g / l)$ are respectively the concentration of thymol in water and oil bulk, $C^{\prime}{ }_{w}(g / l)$ and $C^{\prime}{ }_{o}(g / l)$ are respectively the concentration of thymol at the interface on water and oil side, $K_{w}(\mathrm{~m} / \mathrm{s})$ and $K_{o}(\mathrm{~m} / \mathrm{s})$ are respectively the global mass transfer coefficient of thymol in water and oil, $V_{w}\left(m^{3}\right)$ and $V_{o}\left(m^{3}\right)$ are respectively the water and oil volume and $J\left(\mathrm{~g} / \mathrm{m}^{3} \mathrm{~s}\right)$ is the diffusive flux. The partition coefficient of thymol 
Table 1. Main geometrical parameters of the experiment.

\begin{tabular}{llllll}
\hline Length of ladle & Injection diameter & Height of water bath & Height of oil layer & Volume of water & Volume of oil \\
\hline $\mathbf{L}_{\mathbf{x}}(\mathbf{m})$ & $\mathbf{d}_{\mathbf{i n j}}(\mathbf{m})$ & $\mathbf{h}_{\mathbf{w}}(\mathbf{m})$ & $\mathbf{h}_{\mathbf{o}}(\mathbf{m})$ & $\mathbf{V}_{\mathbf{w}}(\mathbf{l})$ & $\mathbf{V}_{\mathbf{o}}(\mathbf{l})$ \\
\hline 0.27 & $\begin{array}{l}2.5 \times 10^{-3} \\
\text { and } \\
7.9 \times 10^{-3}\end{array}$ & 0.2 & $7.0 \times 10^{-3}$ & 14.6 & 0.49 \\
& & & & \\
\hline
\end{tabular}

Table 2. Physical properties of fluids used in our water experiment.

\begin{tabular}{|c|c|c|c|c|c|}
\hline & Density & Dynamic viscosity & Surface tension & Interfacial tension & Surface tension \\
\hline Fluids & $\boldsymbol{\rho}\left(\mathrm{kg} \cdot \mathbf{m}^{-3}\right)$ & $\mu(\mathbf{P a} \mathbf{s})$ & $\sigma_{\mathrm{a} / \mathbf{w}}\left(\mathbf{N} \cdot \mathbf{m}^{-1}\right)$ & $\sigma_{\mathbf{w} / \mathbf{o}}\left(\mathbf{N} \cdot \mathbf{m}^{-1}\right)$ & $\sigma_{\mathbf{o} / \mathbf{a}}\left(\mathbf{N} \cdot \mathbf{m}^{-1}\right)$ \\
\hline Air & 1.225 & $1.85 \times 10^{-5}$ & $7.12 \times 10^{-2}$ & & \\
\hline Water & 998 & $1.00 \times 10^{-3}$ & & $2.55 \times 10^{-2}$ & \\
\hline Oil & 920 & $7.9 \times 10^{-2}$ & & & $3.17 \times 10^{-2}$ \\
\hline
\end{tabular}

comparing the solubilities of thymol in water and oil at equilibrium is given by

$$
P=\frac{C^{\prime}{ }_{o}}{C^{\prime}{ }_{w}}
$$

Using mass balance for thymol between water and oil from (5) and (6) we can write

$$
\frac{V_{w} d C_{w}}{\mathrm{~d} t}+\frac{V_{o} d C_{o}}{\mathrm{~d} t}=0
$$

After time integration we can write

$$
V_{w} C_{w}+V_{o} C_{o}=V_{w} C_{w, o}
$$

where $C_{w, o}(g / l)$ is the initial concentration of thymol in the water phase bulk. Using (8) and (9) we can rewrite (5) and (6)

$$
\begin{gathered}
\frac{V_{o}}{V_{w}} \frac{d}{\mathrm{~d} t}\left[\frac{C_{o}}{C_{w, o}}\right]=-\frac{1}{\tau_{w}}\left(1-\frac{V_{o} C_{o}}{V_{w} C_{w, o}}\right)+\frac{C^{\prime}{ }_{o}}{\tau_{w} P C_{w, o}} \\
\frac{\tau_{o}}{\tau_{w} P} \frac{d}{\mathrm{~d} t}\left[\frac{C_{o}}{C_{w, o}}\right]=\frac{C^{\prime}{ }_{o}}{\tau_{w} P C_{w, o}}-\frac{C_{o}}{\tau_{w} P C_{w, o}}
\end{gathered}
$$

with $\tau_{w}=V_{w} / K_{w} A$ and $\tau_{o}=V_{o} / K_{o} A$, combining (10) and (11) we can obtain the following differential equation

$$
\frac{d}{\mathrm{~d} t}\left[\frac{C_{o}}{C_{w, o}}\right]=-\frac{1}{\tau_{w}} \frac{1+\beta}{1+\alpha \beta} \frac{C_{o}}{C_{w, o}}+\frac{1}{\tau_{w}} \frac{V_{w} / V_{o}}{1+\alpha \beta}
$$

with $\alpha=K_{w} / P K_{o}$ a case-specific constant assuming $K_{w}$ and $K_{o}$ are time independent. $\beta=V_{w} / V_{o} P$ is a constant depending only on the fluid quantity and nature. Considering that at $t=0$ we know that $C_{o, 0}=0 \mathrm{~g} / \mathrm{l}$, we obtain the following solution for the evolution of concentration of thymol in the oil phase

$$
C_{o}=\frac{V_{w}}{V_{o}} \frac{C_{w, 0}}{1+\beta}\left(1-e^{-\lambda t}\right)
$$

with $\lambda=-\frac{1}{\tau_{w}} \frac{1+\beta}{1+\alpha \beta}$. Using (9) and (13) we can obtain the evolution of concentration of thymol in the water phase

$$
C_{w}=C_{w, 0}-\frac{C_{w, 0}}{1+\beta}\left(1-e^{-\lambda t}\right)
$$

Following (14), we can see that the concentration of thymol follows an exponential decay from the initial value $C_{w, 0}$ to an equilibrium value of $C_{w, 0}-C_{w, 0} /(1+\beta)$. The characteristic concentration decay time is given by

$$
\begin{gathered}
\tau_{c}=-1 / \lambda \\
\tau_{c}=\tau_{w} \frac{1+\alpha \beta}{1+\beta}
\end{gathered}
$$

$\alpha$ and $\beta$ need to be small to have a minimum concentration decay time around $\tau_{c, \min } \sim \tau_{w}$. It is generally acknowledged that in an industrial ladle the desulphurization reaction of liquid steel with slag has a high $P K_{o}$ value. Then, the steel phase mass transfer is controlled by the steel phase mass transfer resistance. In the case of water experiment, thymol has a high partition coefficient between oil and water $(P>350)$. Assuming that $K_{o}$ is not too small it leads to a mass transfer controlled by the water phase resistance. 
Now, if we take the logarithm of (14) and use the expression of $\lambda$ we can write

$$
\frac{1}{\tau_{w}} \frac{1+\beta}{1+\alpha \beta} t=\ln \left[\frac{C_{w}}{C_{w, 0}}(1+\beta)-\beta\right]
$$

In reference [2], the authors assume that $\alpha \ll 1 / \beta$ is verified in the mass transfer configuration associated with the desulfurization process. Considering the same assumption in our study, we can rewrite (15) to obtain

$$
\frac{K_{w} A}{V_{w}} t=\frac{\ln \left[\frac{C_{w}}{C_{w, 0}}(1+\beta)-\beta\right]}{1+\beta}
$$

In the expression above, the product $K_{w} A / V_{w}$ is called the capacity coefficient. From relation (16), it is possible to estimate directly the capacity coefficient from the measured thymol concentration in water on the righthand side of (16). This formulation is used to determine the global mass transfer coefficient in the following experimental results.

\subsection{Numerical model}

To resolve the problem, we solve numerically the partial differential equations with the open-source Free Software library Basilisk. Basilisk allows to perform Direct Numerical Simulation or DNS, meaning there are no turbulence models as the Navier-Stokes equations are resolved down to the smallest scales. Basilisk has the possibility to adapt the mesh dynamically following a quadtree/octree structure. Typically, the minimum mesh size $\Delta_{\text {min }}$ can be computed from the maximum level of refinement maxlevel which defines the maximum number of cells in one direction and the size of the domain $L_{0}$ with $\Delta_{\min }=L_{0} / 2^{\text {maxlevel }}$. The numerical schemes used in Basilisk are based on its ancestor the Gerris solver and can be found in [16,17]. The flow is described with the incompressible Navier-Stokes equations with surface tension term treated by a Continuous Surface Force [18] which can be written

$$
\begin{aligned}
& \rho\left(\frac{\partial \boldsymbol{u}}{\partial t}+\boldsymbol{u} \cdot \nabla \boldsymbol{u}\right)=-\nabla p+\nabla \cdot(2 \mu \boldsymbol{D})+\sigma k \boldsymbol{n} \delta_{S} \\
& \nabla \cdot \boldsymbol{u}=0
\end{aligned}
$$

with $\boldsymbol{D}=\left(\nabla \boldsymbol{u}+(\nabla \boldsymbol{u})^{T}\right) / 2$ the deformation tensor. Because we are dealing with multiphase flow the variations of density and viscosity inside the domain are described with the help of the Volume of Fluid method. The volume fraction $\chi(x, t)$ is described by

$$
\chi(x, t)=\left\{\begin{array}{l}
1 \text { inside fluid phase } \\
0<\chi<1 \text { at interface } \\
0 \text { elsewhere }
\end{array}\right.
$$

which obeys to an advection equation

$$
\frac{\partial \chi}{\partial t}+\nabla \cdot(\chi \boldsymbol{u})=0
$$

Because we are in presence of a three-phase flow, we cannot use the implicit declaration of phase two as in a twophase flow. Instead, we declare explicitly three volume fractions corresponding to each phase. To ensure that a cell is not filled with more than one phase the sum of $n$ volume fractions should respect

$$
0 \leq \sum_{k=1}^{n} \chi_{k}(x, t) \leq 1
$$

To do so at each time-step we normalize the volume fractions $\chi_{k}$ of the $k-t h$ phase with the sum of volume fractions

$$
\chi_{k, n o r m}(x, t)=\frac{\chi_{k}(x, t)}{\sum_{k=1}^{n} \chi_{k}(x, t)}
$$

This way we can follow a one fluid description with variable density and viscosity determined in the domain with arithmetic mean

$$
\begin{aligned}
& \rho(\chi) \equiv \chi_{a} \rho_{a}+\chi_{w} \rho_{w}+\chi_{o} \rho_{o}, \\
& \mu(\chi) \equiv \chi_{a} \mu_{a}+\chi_{w} \mu_{w}+\chi_{o} \mu_{o},
\end{aligned}
$$

Surface tension is acting on the interface between two fluids, but here there is more than one possibility of fluid neighbor for one phase. That is why we decompose the physical surface tension into phase-specific surface tension just depending on the phase and not the fluid in contact following $[19,20]$

$$
\begin{aligned}
& \sigma_{w} \equiv\left(-\sigma_{o / a}+\sigma_{a / w}+\sigma_{w / o}\right) / 2 \\
& \sigma_{o} \equiv\left(\sigma_{o / a}-\sigma_{a / w}+\sigma_{w / o}\right) / 2 \\
& \sigma_{a} \equiv\left(\sigma_{o / a}+\sigma_{a / w}-\sigma_{w / o}\right) / 2
\end{aligned}
$$

To take into account the concentration variation we need to consider also the generic scalar transport equation of concentration $c$ (amount of chemical species/unit volume) for an incompressible flow and without sources or sinks

$$
\frac{\partial c}{\partial t}=\nabla \cdot(D \nabla c)-\nabla \cdot(\mathrm{uc})
$$

We can separate (23) in two parts: $-\nabla \cdot(D \nabla c)$ describes the molecular diffusion of $c$; $-\nabla \cdot(u c)$ describes convection of $c$ by the flow.

$c$ has no influence on the velocity field determined by solving the Navier-Stokes equation and is only used to consider diffusion, therefore it is a passive scalar or tracer. We solve the two parts of (23) in two distinct steps and start by solving the convection part. To do so, we define a $\mathrm{k}$-th-phase-specific concentration following

$$
T_{k}=c \chi_{k}
$$

$T_{k}$ is confined to a VOF-determined phase in the same manner as described in [21]. The method in the latter reference ensures that the tracer $c$ cannot cross a VOF 
Table 3. Main dimensionless characteristic of the steel and the slag equivalent phase for our experiment and the experiment of [23-25].

\begin{tabular}{llllll}
\hline & Fluids & $\mathbf{m}$ & $r$ & $\mathbf{h}_{\mathbf{m}} / \mathbf{L}_{\mathbf{x}}$ & $\mathbf{h}_{\mathbf{s}} / \mathbf{h}_{\mathbf{m}}$ \\
\hline Our experiment & Water-oil mixture & $1.3 \times 10^{-2}$ & 1.09 & 0.74 & 0.035 \\
Krishnapisharody et al. [24] & Water-paraffin oil & $1.5 \times 10^{-2}$ & 1.15 & 0.5 & 0.048 \\
Krishnapisharody et al. [24] & Water-motor oil & $3.4 \times 10^{-2}$ & 1.14 & 0.5 & 0.048 \\
Yonezawa et al. [23] & Mercury-silicon oil & $3.2 \times 10^{-2}$ & 14.2 & 0.78 & 0.044 \\
Yonezawa et al. [23] & Steel-slag & $5.3 \times 10^{-2}$ & 2.43 & 0.80 & 0.014 \\
Thunman et al. [25] & Ga,In,Sn-glycerol & $4.0 \times 10^{-2}$ & 4.89 & 0.72 & 0.100 \\
\hline
\end{tabular}

interface and limits the unwanted numerical diffusion. Therefore, instead of directly doing the convection of $c$ we do it on $T_{k}$.

Before considering the diffusion of the tracer $c$ computed from (24), we need to consider the concentration jump in the interfacial cells. Because of the formulations used in the Basilisk code, we could not easily add the equivalent of the partition ratio $P$ at the interface. We use instead a Dirichlet condition imposed on the $k$ th phase side

$$
\text { if } \chi_{k}<0.5, T_{k}=0
$$

This is an important assumption made to approximate the diffusion in the configuration of interest. Within this assumption it is not possible to ensure that the absolute diffusive flux of tracer leaving one phase stays constant when entering another phase.

Then we solve the second part of (23) which is the molecular diffusion of $c$. Once the tracer $c$ has diffused, the value of $T_{k}$ is updated with the new value of $c$ using (24).

Finally, to simplify the model we consider constant phase-specific diffusion coefficients in the water and oil phase. In the air phase we set the diffusion coefficient to $D_{a}=0 \mathrm{~m}^{2} / \mathrm{s}$ as we neglect its influence on the mass transfer.

To have an idea of the smallest scales to be resolved in the simulation we can use the Kolmogorov length scale given by

$$
\eta=\left(\frac{v_{w}^{3}}{\in / \rho_{w}}\right)^{1 / 4}
$$

Using an approximation of the kinetic energy dissipation in the water experiment configuration we can directly compute (26) from the gas flow rate value with

$$
\eta=\left(\frac{v_{w}^{3}}{g Q / L_{x}^{2}}\right)^{1 / 4}
$$

At the lowest air flow rate considered in our experiment $Q=0.6 \mathrm{l} / \mathrm{min}$ it gives $\eta=165 \mu \mathrm{m}$.

In reference [22], the authors postulated a criterion to determine the minimum mesh size needed to resolve the Kolmogorov length scale in a DNS $\Delta_{\min } \leq 2.1 \eta$. As we are interested about mass transfer, we need to consider the Schmidt number $S c=\mu / \rho D$ characterizing the ratio of momentum diffusivity and mass diffusivity. In the experiment the Schmidt number of thymol in water is $S c_{t h, w}=1.48 \times 10^{3}$. The higher is the Schmidt number, the smaller is the concentration boundary layer. To avoid the use of an extremely small mesh size we choose to do simulation with smaller Schmidt number than $S c_{t h, w}$ and consider four tracers in the water phase in the range of $S c_{w} \in\{1,4,10,40\}$. We also consider four tracers in the oil phase but at higher Schmidt number than in the water phase because of the difference in the viscosity and density. The range in the oil phase is $S c_{o} \in\{10,40,100,400\}$. Then we will extrapolate the numerical Sherwood number values to the experimental Schmidt number. At low value of Schmidt number, the concentration boundary layer thickness is approximately equal to the hydrodynamic boundary layer thickness. If we consider a minimum mesh size of $\Delta_{\min }=104.4 \mu \mathrm{m}$ corresponding approximately to a maximum mesh resolution of $2^{11}$ cells in one direction. At this mesh resolution we can describe the initial oil layer with a maximum of 50 cells. Also, if we consider an oil droplet of $3 \mathrm{~mm}$ of diameter with the same mesh resolution we end up with 22 cells in its diameter.

\section{Results}

In the following, for easier comparison between experiments using other fluids than water and oil we will denote the steel and the slag equivalent phase respectively with the indices $m$ and $s$. We also have to mention that we have used in the numerical simulation a reference value for the surface tension value of $\sigma_{a / w}=72 \times 10^{-3} \mathrm{~N} / \mathrm{m}$ instead of the measured value of Table 2 .

\subsection{Hydrodynamic characterization}

\subsubsection{Experimental results}

In Table 3 we compute the viscosity ratio $m=\mu_{m} / \mu_{s}$, the density ratio $r=\rho_{m} / \rho_{s}$ together with the bath height ratio $h_{m} / L_{x}$ and the slag height ratio $h_{s} / h_{m}$. We can see from Table 3 that the viscosity ratio is close to the liquid steelslag value of the industrial measurement of [23] for almost all the experiments except the one of [24] using motor oil. Now, if we look at the density ratio, we can see that the 


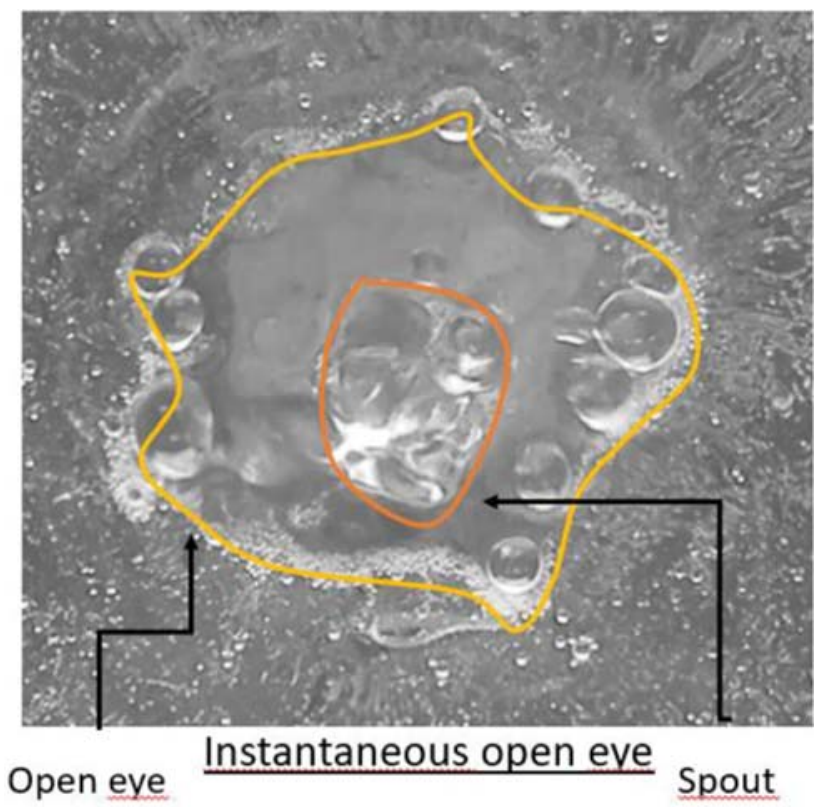

Fig. 2. Top view of the instantaneous experimental open eye obtained at $Q=0.6 \mathrm{l} / \mathrm{min}$. The color code is in yellow: open eye contour; orange: spout zone.

water experiments present a lower density ratio compared to the liquid metal experiments. If we look now at the bath height ratio, we can see that in all the experiments, it is mostly around 0.75 except for the experiment of [24]. Finally, for the slag height ratio, we can see that in most of the experiments the value is not far from the industrial configuration corresponding to a thin slag layer.

Images of the top surface of the water experiment are recorded with a video camera making possible to follow the deformation of the oil layer under the influence of gas injection. In Figure 2 showing an instantaneous image of the top view of the water experiment, we can identify the contour of the open eye of the oil layer with a yellow line and the bubbles spout contour in orange. Unfortunately, it has not been possible to automatically plot the open eye contour of all the images for each air flow rate. So, we choose to rather plot manually the open eye contour for four frames separated by approximately $1.8 \mathrm{~s}$ for each air flow rate. Then, these plots have been postprocessed using Image J to determine the experimental time average open eye area and its standard deviation.

In Figure 3 we plot the dimensionless open eye area as a function of the Froude number. From Figure 3, first we can note that most of the water experiments with cross symbols show similar evolution of the open eye area as a function of the Froude number. On the other hand, there is more dispersion between the liquid metal experiments. Indeed, we observe for the liquid metal experiments a smaller slope for the evolution of the dimensionless open eye area than for the water experiments. If we look at the industrial results of [23] (filled red symbols in Fig. 3), we can see that the latter are closer to the water experiments than the cold liquid metal experiments of $[23,25]$. This may be due to the very high value of $r$ and in the case of [22] of $h_{s} / h_{m}$ in the cold

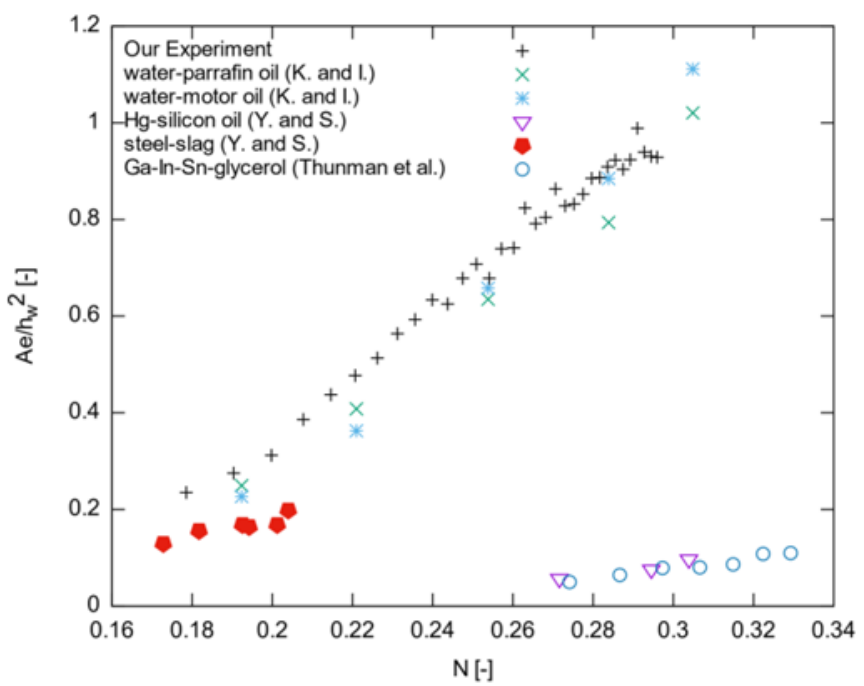

Fig. 3. Ratio of time average open eye area with the square of the bath height as a function of the Froude number for our experiment and the experiments of Krishnapisharody et al. [24], Yonezawa and Schwerdtfeger [23] and Thunman et al. [25].

liquid metal experiments. As a result, it is likely that the implicit correlation in Figure 3

$$
\frac{A_{e}}{h_{w}^{2}}=f(N)
$$

should be replaced by

$$
\frac{A_{e}}{h_{w}^{2}}=f\left(N, r, \frac{h_{s}}{h_{m}}\right)
$$

the exact form of the function $f$ would still require some investigations. To conclude, the literature shows that the open eye area is very strongly affected by the fluid density ratio and perhaps the ratio of heights $h_{s} / h_{m}$. Extrapolation from the experimental water/oil configuration to the industrial liquid-steel/slag configuration should take this into account.

\subsubsection{Numerical results}

To capture the open eye numerically first we do simulations of the water experiment configuration. Then we postprocess the generated images of the top view of the oil interface with the software ImageJ to get the open eye area. From Figure 4 we can make a clear distinction from the oil interface colored in red and the other phase in blue. Taking advantage of this we could obtain this time the temporal evolution of the open eye area from all the images of the numerical simulations.

In Figure 5, we compare the time evolution of the open eye area obtained for different air flow rates with $d_{i n j}=7.9 \mathrm{~mm}$. First, it is important to mention that the data gap observed for $Q=1.5 \mathrm{l} / \mathrm{min}$ is due to a format problem of the generated images and is not interpolated from the non-missing data. We can see that globally the 


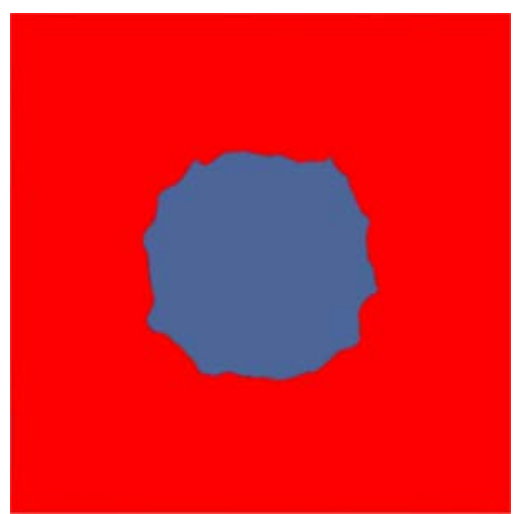

Fig. 4. Top view of the instantaneous numerical open eye, obtained at $Q=0.6 \mathrm{l} / \mathrm{min}$. The numerical results are obtained with a minimum grid size of $\Delta x=527 \mu \mathrm{m}$ and the oil interface is colored in red.

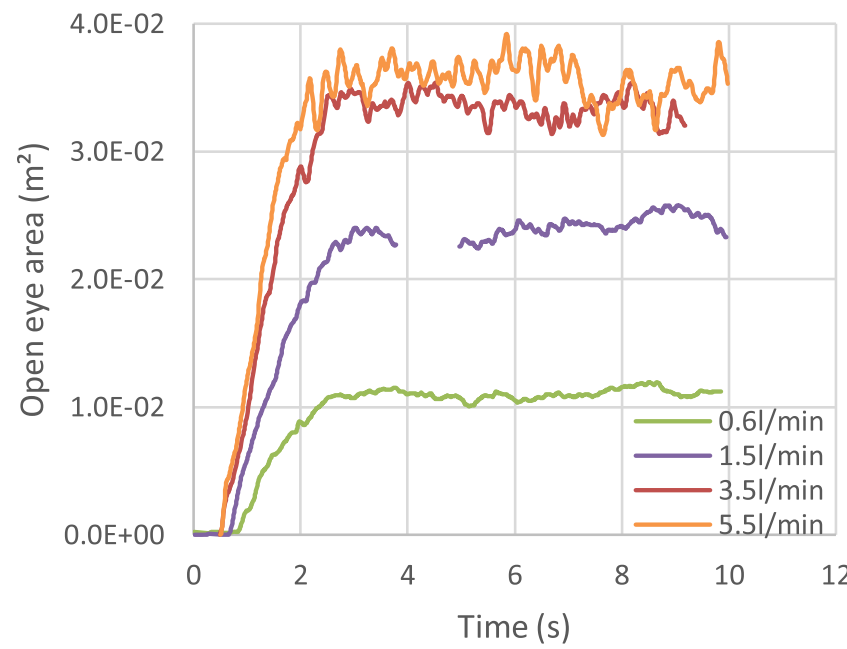

Fig. 5. Time evolution of the open eye area for different air flow rates with $d_{i n j}=7.9 \mathrm{~mm}$. The results are obtained with a minimum grid size $\Delta_{\min }=527 \mu \mathrm{m}$ corresponding to a maxlevel $=9$ of adaptive refinement.

open eye area follows the increase of the air flow rate and that waiting ten seconds of physical time seems to be enough to reach a statistical steady state. If we look at the data for $Q<3.5 \mathrm{l} / \mathrm{min}$ we can see an important change in the slope during the transient regime and on the asymptotic value of the open eye area when the air flow rate is increased. Looking now at results for $Q \geq 3.5 \mathrm{l} / \mathrm{min}$ we observe mostly difference on the fluctuations between $Q=3.5 \mathrm{l} / \mathrm{min}$ and $Q=5.5 \mathrm{l} / \mathrm{min}$. We can note that the convergence of the open eye area toward an asymptotic value is slower for high air flow rate than for low air flow rate.

We can see on Figure 6 that the numerical simulation reproduces qualitatively well the same flow behavior as the one that will be seen later on Figure 7 . We can identify a central bubble plume generating an open eye in the oil layer when it reaches the free surface. The oil layer is relatively

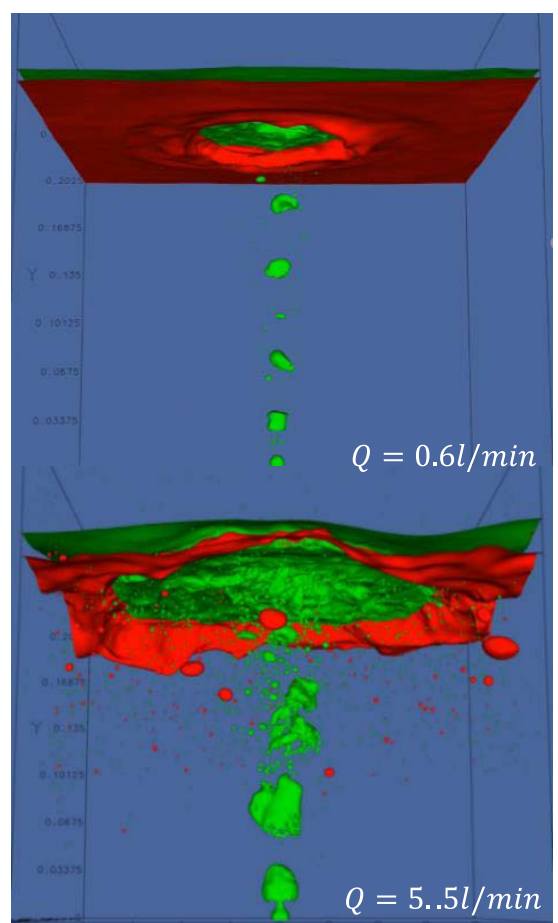

Fig. 6. Front view of the simulation with $d_{i n j}=7.9 \mathrm{~mm}$ with a minimum grid size $\Delta_{\min }=527 \mu \mathrm{m}$. The air interface is colored in green, and the oil interface is colored in red.

stable, and no oil droplet is observed at low air flow rate (cf. Fig. 6 top picture). While the free surface is highly perturbated at high air flow rate (cf. Fig. 6 bottom picture) and some oil droplets of various sizes can be identified in the water phase.

\subsubsection{Comparison of the results}

In Figure 8 we plot the time-averaged numerical and experimental open eye area. First, we can see that the increase of the open eye area with flow rate can be well described by a logarithmic function of the flow rate in both cases. Furthermore, we can note that the numerical results overestimate the experimental open eye area, and that this tendency is more pronounced at low air flow rate than at high air flow rate. We have searched unsuccessfully for an explanation of the discrepancy.

One possibility is that the spatial discretization error is still large. We have compared $\Delta_{\min }=527 \mu \mathrm{m}$ and $\Delta_{\min }=$ $264 \mu \mathrm{m}$ simulations without noticing an improvement when the grid is refined but it is possible that very thin oil layers form because of the positivity of the spreading coefficient

$$
S=\sigma_{a / w}-\left(\sigma_{o / a}+\sigma_{w / o}\right)
$$

Since $S=0.0148 N / m>0$, oil spreads in thin layers [26]. These layers could be much thinner than even the smallest grid size used, so that even finer grids would be needed. 


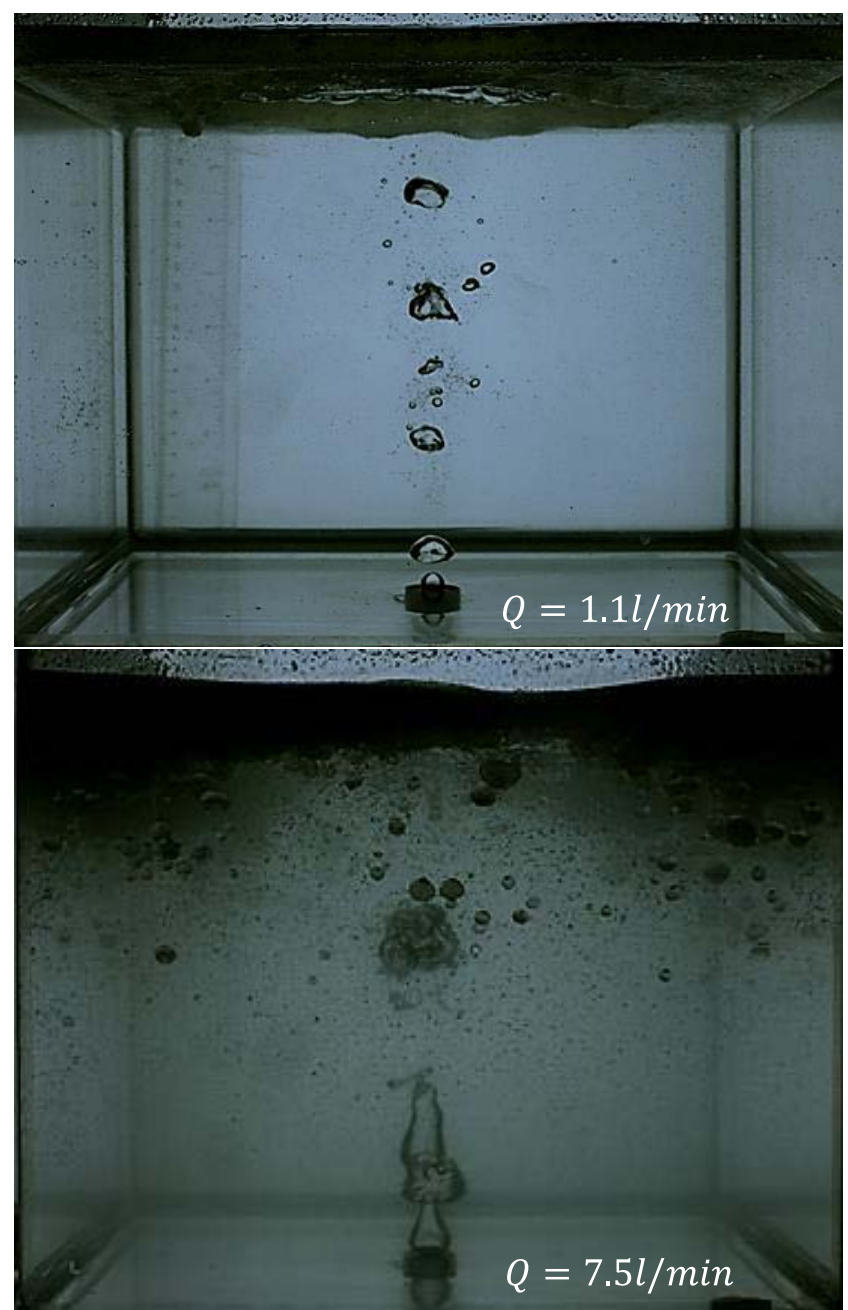

Fig. 7. Front view picture of the water experiment with thymol dissolved in the water obtained with $\mathrm{d}_{i n j}=7.9 \mathrm{~mm}$.

This would explain the discrepancy between numerics and experiment.

\subsection{Mass transfer characterization}

\subsubsection{Experimental results}

From the time evolution of the concentration of thymol in water (cf. Fig. 9, top picture), we can observe a good fit of the measurement with an exponential decay in agreement with correlation (14). Now, from the concentration measurement we can plot minus the right-hand side of (16) in Figure 9 bottom picture. Taking the slope of a linear fit of the curve, we can compute the product of the time average mass transfer coefficient in the water with the interfacial area or capacity coefficient.

From the results of Figure 9 bottom picture, the slope of the linear fit is $6.2 \times 10^{-5}$ so, at this air flow rate it gives a capacity coefficient of $K_{w} A / V_{w}=6.2 \times 10^{-5} \mathrm{~s}^{-1}$.

We repeat this process for different air flow rates and different injection diameters in Figure 10. From our experimental results in Figure 10 first we can observe that

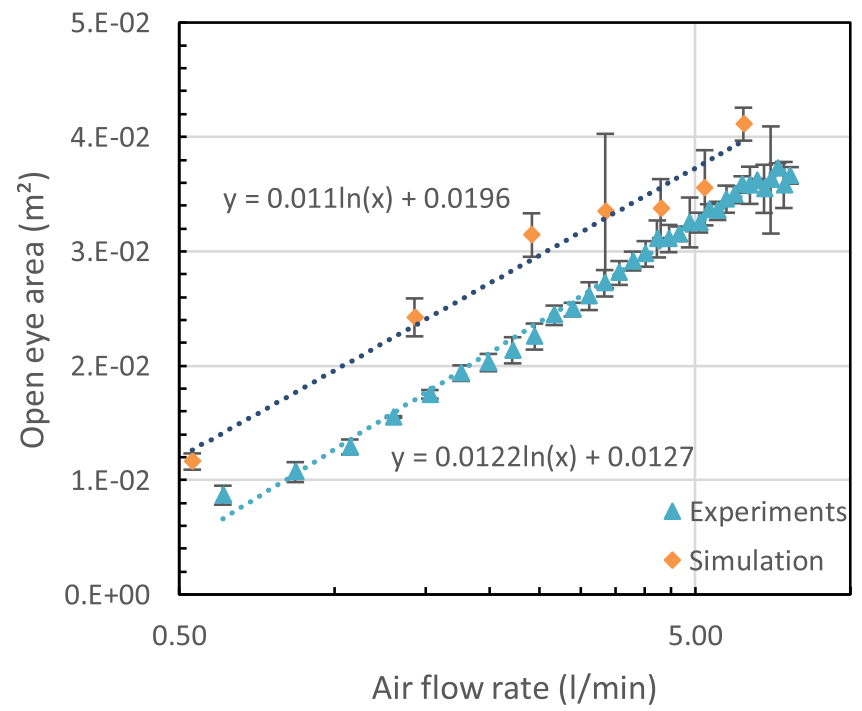

Fig. 8. Evolution of the time averaged open eye area of several experiments with its standard deviation and numerical timeaveraged open-eye area with its temporal standard deviation as a function of the air flow rate obtained with $\mathrm{d}_{i n j}=7.9 \mathrm{~mm}$. The simulation results are obtained with a minimum grid size of $\mathrm{D}_{\min }=527 \mathrm{~mm}$ corresponding to a maxlevel $=9$ of adaptive refinement.

the capacity coefficient increases when the air flow rate is increased for both injection diameters. Even if we have less data for the $d_{i n j}=2.35 \mathrm{~mm}$ case it appears that capacity coefficient varies only a little compared to the $d_{i n j}=7.9 \mathrm{~mm}$ case. We can also identify two different mass transfer regimes below and above a critical air flow rate $Q_{c} \sim 5.0 \mathrm{l} /$ min for both injection diameters. Secondly, if we compare our results with the one obtained by [2], we observe a similar evolution with a critical air flow rate qualitatively the same as the one we measured with $d_{i n j}=7.9 \mathrm{~mm}$. However, the slope of the first mass transfer regime is higher in the results of [2] than in our results. For the second mass transfer regime similar slope is observed between our results and the results of [2].

In reference [2] the authors justify the change of mass transfer regime observed in Figure 10 by the formation of oil droplets of various sizes at high air flow rate. In the top picture of Figure $7(Q=1.1 \mathrm{l} / \mathrm{min})$ no oil droplet is visible in the water. At higher air flow rates, oil droplets appear transiently a few minutes after the start of air injection, then disappear probably by flotation to the top oil layer. If we increase the air flow rate further, a lot of dark spots which correspond to oil droplets can be identified (see the bottom picture of Figure 7 for $Q=7.5 \mathrm{l} / \mathrm{min}$ ). Indeed, oil droplets of various sizes and shapes are present in water both at the start of air injection and after three hours of agitation at this high air flow rate. These oil droplets increase the interfacial area between water and oil resulting in an abrupt increase of mass transfer.

Now, to compare our results with results in the literature we compute the time average Sherwood number which is the ratio of convective mass transfer to the rate of 

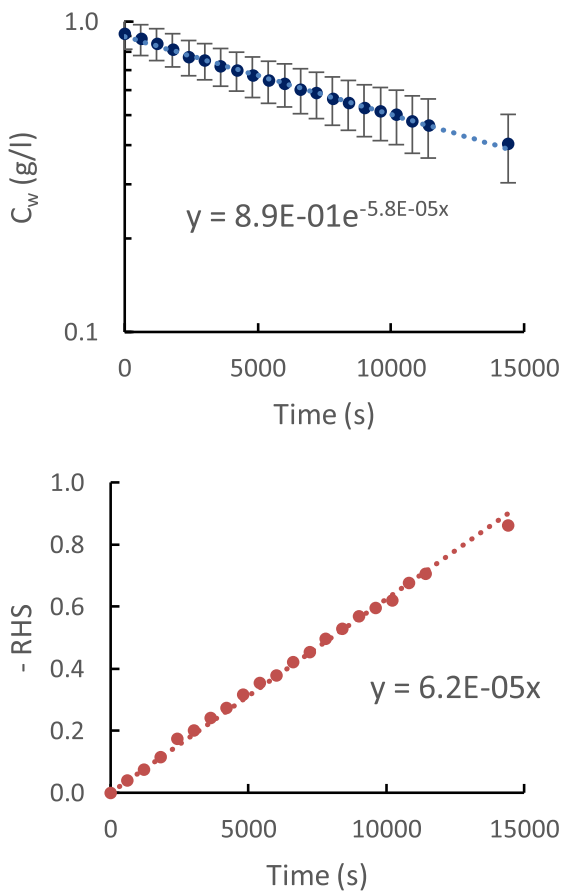

Fig. 9. Top: Time evolution of the concentration of thymol in water at $\mathrm{Q}=2.5 \mathrm{l} / \mathrm{min}$ and $\mathrm{d}_{i n j}=7.9 \mathrm{~mm}$. The error bars correspond to the measurement errors (cf. Supplementary Material: Fig. 1). Bottom: Time evolution of minus the righthand side of (16) obtained with $\mathrm{Q}=2.5 \mathrm{l} / \mathrm{min}$ and $\mathrm{d}_{i n j}=7.9 \mathrm{~mm}$.

diffusive mass transport. We consider as characteristic length scale $L_{c}=L_{x} / 4$, (the $1 / 4$ factor considers that boundary layers develop on the side of the open eye overs distances shorter than $L_{x}$ ) then we can define

$$
S h_{m}=\frac{K_{m} L_{x}}{4 D_{w}}
$$

Figure 11 shows a comparison of the time average Sherwood number for water experiments (our results with $d_{i n j}=7.9 \mathrm{~mm}$ and the one of [2]) and liquid metal experiments [3,6]. From the results of Figure 11, we observe for each experiments an abrupt increase of the mass transfer corresponding to the start of the second mass transfer regime. The results of [6] show a higher value of the Sherwood number than the other experiments with a difference of a factor ten with the results of [3]. This is interesting to note from Table 4 that these two experiments are done with similar Schmidt number in the steel equivalent phase. Now for the water experiments, if we look back at Figure 10 we see that there is a small difference in the value of the capacity coefficient between our results and the one of [2]. If we assume that these two experiments follow the same chemical reaction between water and oil, it means that the difference observed in Figure 11 is mainly due to the difference in the size of the ladle. The same goes for the difference in the Sherwood number for the liquid metal experiments of $[3,6]$. Beside the difference in the

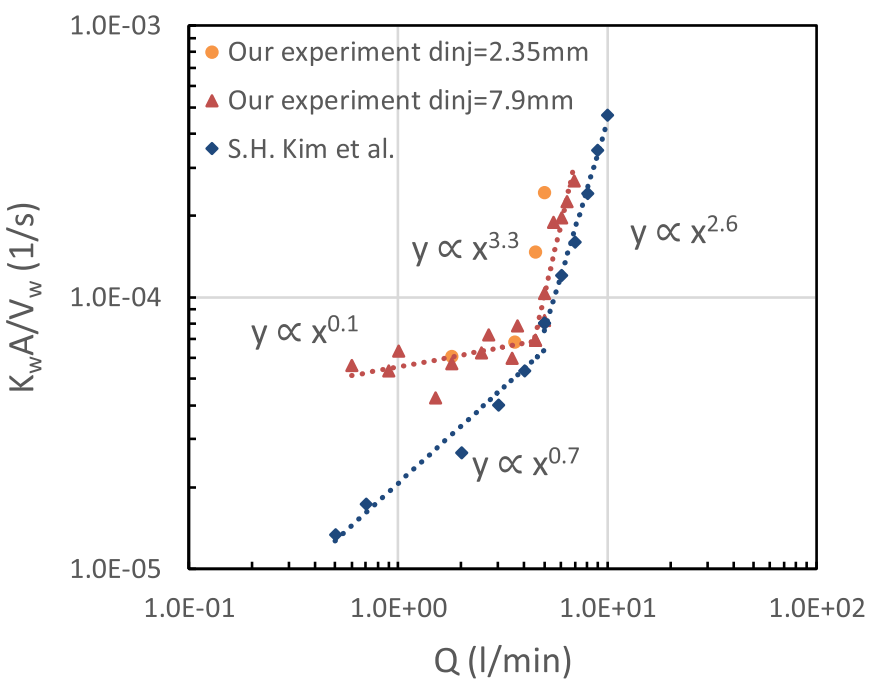

Fig. 10. Evolution of the capacity coefficient or product of the time average mass transfer coefficient with the interfacial area as a function of gas flow rate for our experiment and the one of Kim et al. [2].

Sherwood number value, we can see that the liquid metal experiments also show a smaller slope for the second mass transfer regime than for the water experiments.

\subsubsection{Numerical results}

We perform simulations for five distinct air flow rates below and around the mass transfer transition observed experimentally. The simulations are started after $t=7.6 \mathrm{~s}$ of physical time with a minimum grid size of $\Delta_{\min }=527 \mu \mathrm{m}$ corresponding to a maxlevel $=9$ of adaptive refinement. Then when the mass transfer transitory regime is finished, we use the obtained results to restart the simulation with half the minimum grid size corresponding to a maxlevel= $n+1$ of adaptive refinement. This allows us to save computational time by not recomputing the mass transfer transitory regime for each mesh resolution. First, we consider a case with a constant air flow rate of $Q=0.6 \mathrm{l} /$ min and various mesh resolutions. On Figure 12 we observe a slow decrease of the normalized concentration of tracer in water. The decrease over the eight seconds of simulation is small $(2 \%)$ due to the limited simulation time. When the mesh resolution increases from a maxlevel $=9$ to $\operatorname{maxle-}$ $v e l=10$ of adaptive refinement we can see an important change in the slope of the normalized concentration. We remark that the change of the slope is smaller when increasing the maxlevel of adaptive refinement from maxlevel $=10$ to $\operatorname{maxlevel}=11$. This indicates that at $S c=10$ and $Q=0.6 \mathrm{l} / \mathrm{min}$ we start to obtain mesh convergence from a maxlevel $=10$ of adaptive refinement corresponding to a minimum mesh size of $\Delta_{\min }=263.5 \mu \mathrm{m}$.

If we now look at the results of Figure 13 obtained at a higher air flow rate of $Q=3.5 \mathrm{l} / \mathrm{min}$, we observe globally the same behavior than in Figure 12. However, even if we have less data for this high air flow rate, in Figure 13 we can observe some small oscillations on the normalized concentration. It appears also that there is a significative 
Table 4. Main dimensionless physical properties for our experiment and the experiments of $[2,3,6]$.

\begin{tabular}{lllcccccc}
\hline & $m$ & $r$ & $S c_{m}$ & $S c_{s}$ & $P$ & $\beta$ & $h_{m} / L_{x}$ & $h_{s} L_{x}$ \\
\hline Our experiment & 0.01 & 1.1 & $1.5 \times 10^{3}$ & $1.3 \times 10^{7}$ & 350 & $9.0 \times 10^{-2}$ & 0.7 & 0.04 \\
Kim et al. [2] & 0.03 & 1.1 & $1.5 \times 10^{3}$ & $2.4 \times 10^{6}$ & 350 & $9.0 \times 10^{-2}$ & 1.0 & 0.03 \\
Ishida et al. [3] & 0.005 & 2.4 & $1.8 \times 10^{2}$ & $1.3 \times 10^{5}$ & 350 & $1.2 \times 10^{-1}$ & 0.9 & 0.03 \\
Lachmund et al. [6] & 0.005 & 2.4 & $1.8 \times 10^{2}$ & $1.3 \times 10^{5}$ & 350 & 4.6 & 0.9 & 0.03 \\
\hline
\end{tabular}

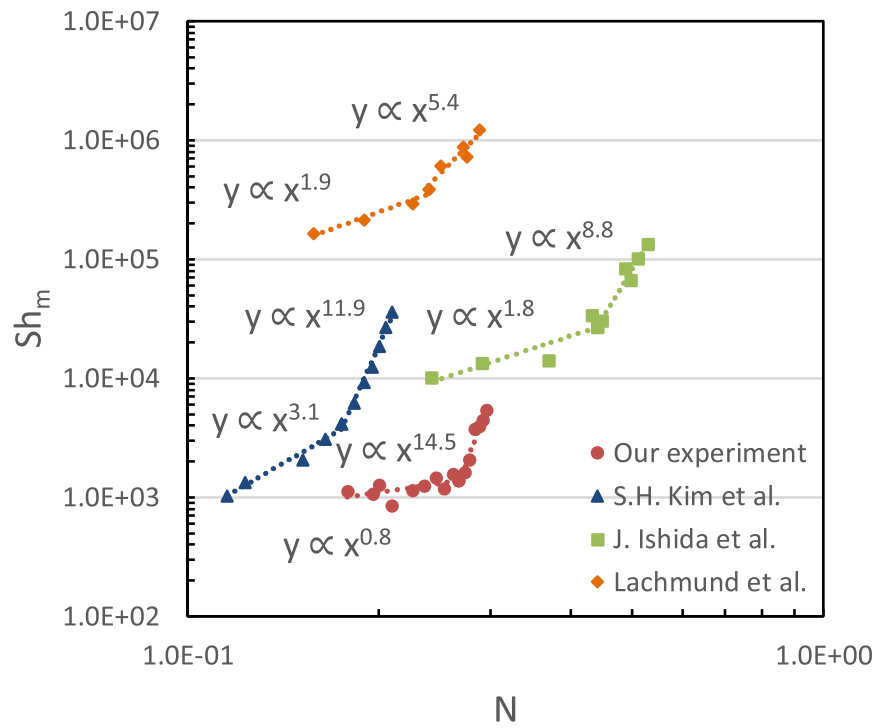

Fig. 11. Time average Sherwood number in the steel equivalent phase as a function of the Froude number for our experiment and the experiment of Kim and Fruehan [2], Lachmund et al. [6] and Ishida et al. [3].

difference of the slope of the normalized concentration when the mesh resolution increases from maxlevel $=10$ to $\operatorname{maxlevel}=11$ of adaptive refinement.

From the time evolution of the concentration of tracer in water, we can compute the mass transfer coefficient in the water assuming that the concentration at the interface is set to zero because of the Dirichlet condition. So, we can write

$$
K_{w, \text { num }}(t) A=\frac{V_{w}}{C_{w, \infty}} \frac{d C_{w}}{\mathrm{~d} t}
$$

As we do not know the experimental oil-water interfacial area, we consider the water-oil area without gas injection as reference area $A=L_{x}^{2}$. From this we can compute the instantaneous numerical Sherwood number using again definition

$$
S h_{w, \text { num }}=\frac{K_{w, n u m}(t) L_{x}}{4 D_{w}}
$$

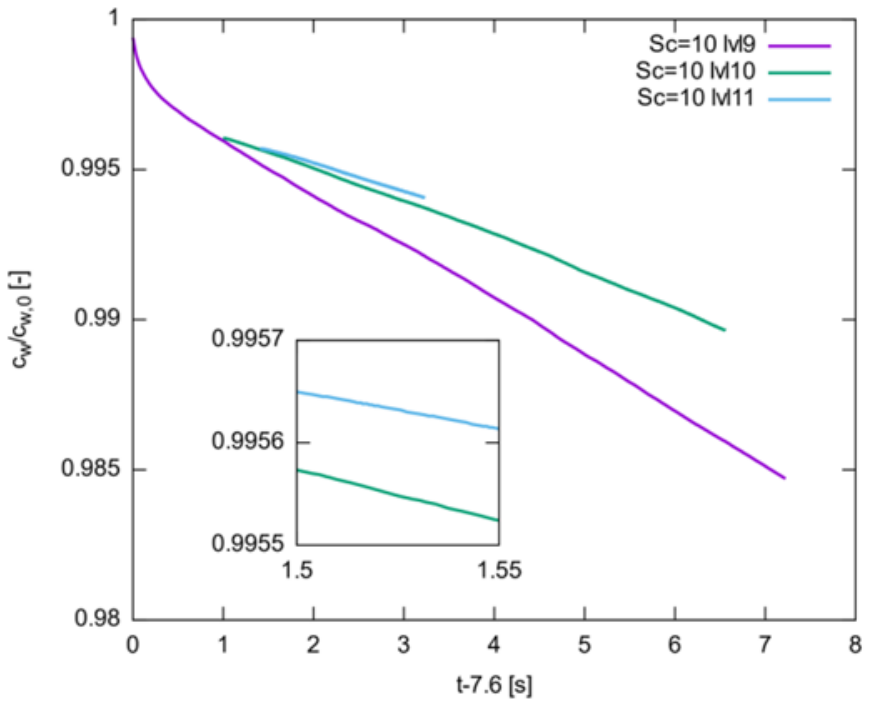

Fig. 12. Time evolution of the normalized concentration of tracer in the water phase $C_{w} / C_{w, o}$ with different mesh resolutions at $S c_{w}=10$ and $Q=0.6 \mathrm{l} / \mathrm{min}$ and with $d_{i n j}=7.9 \mathrm{~mm}$.

In Figure 14 we can observe for a higher mesh resolution than a maxlevel $=9$ a transitory regime where the numerical Sherwood number increases until it reaches an asymptotic value. We can also observe some small fluctuations around an asymptotic value which decrease while the mesh resolution is increased. If we compare the asymptotic value of the numerical Sherwood number $\left(S h_{w, \text { num }}\right)$ for the different mesh resolutions, we can note a higher value of $S h_{w, \text { num }}$ at a low mesh resolution than at a very fine mesh resolution.

Now, if we look at the time evolution of the numerical Sherwood number at $Q=3.5 \mathrm{l} / \mathrm{min}$ in Figure 15 we observe that we have not yet reached an asymptotic value of the Sherwood number for a maxlevel $=11$ of adaptive refinement. We can also remark that in addition to some small fluctuations of the numerical Sherwood number some more important oscillations from an asymptotic value can be seen in Figure 15 considering a maxlevel $<11$. This leads to a higher standard deviation on the fitted value of the asymptotic or time average numerical Sherwood number in Figure 15 than in Figure 14. 


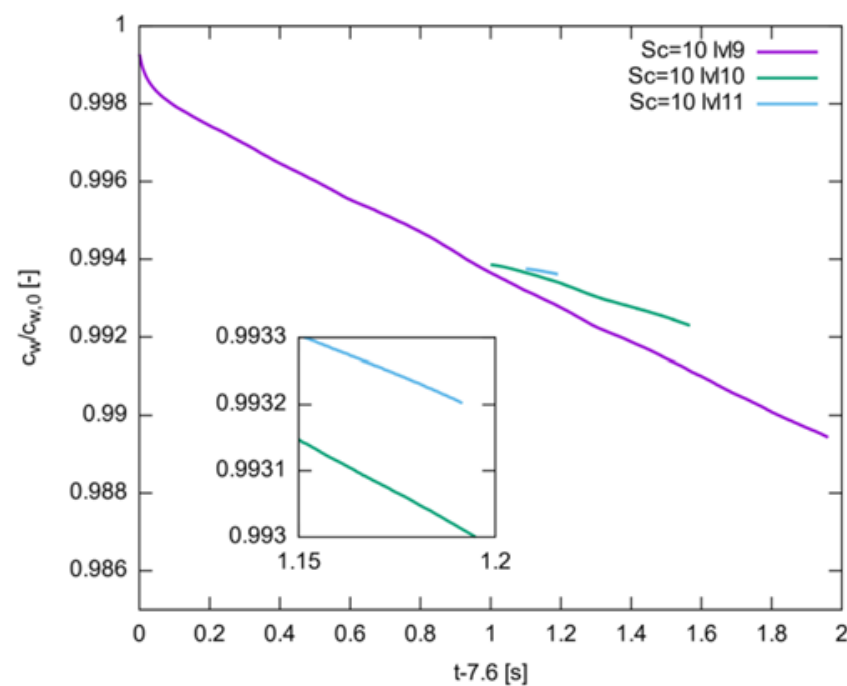

Fig. 13. Time evolution of the normalized concentration of tracer in the water phase $C_{w} / C_{w, 0}$ with different mesh resolutions at $S c_{w}=10$ and $Q=3.5 \mathrm{l} / \mathrm{min}$ and with $d_{i n j}=7.9 \mathrm{~mm}$.

We repeat this process for several Schmidt numbers $S c_{w} \in\{1,4,10,40\}$ and for all the air flow rates $Q \in\{0.6$, $2.5,4.5,5.5,6.5\} \mathrm{l} / \mathrm{min}$. (Some complementary data on the simulations and their results can be found in the Supplementary Material section and in the author's PhD thesis [27]).

We also want to verify the assumption made before that the product of coefficients $\alpha$ and $\beta$ defined above is small. First, we compute the time average numerical mass transfer coefficient from the time-averaged value of the Sherwood number with

$$
K_{k}=\frac{S h_{k, \text { num }} v_{k}}{h_{w} S c_{k}}
$$

Then the ratio of the time averaged mass transfer coefficients ratio between the water and the oil phase is

$$
\frac{K_{w}}{K_{o}}=\frac{S h_{w} v_{w} S c_{o}}{S h_{o} S c_{w} v_{o}}
$$

To compute (35) we need to determine the value of the Sherwood number in the oil phase $S h_{o}$. To do so we proceed in the same way than for the tracer in the water phase by first measuring the time evolution of the concentration of tracer in the oil phase (cf. Fig. 16). We can see from Figure 16 results that at $Q=0.6 \mathrm{l} / \mathrm{min}$ the normalized concentration of tracer in the oil phase decreases more rapidly than in the water phase (cf. Fig. 12). This can be explained by a higher diffusion coefficient in the oil phase due to the difference in the viscosity and density with the water phase. We can also note that almost no difference in the slope of the normalized concentration can be seen. Now, if we look at the Sherwood number in the oil phase in Figure 17 we can see that all the curves converge toward the same asymptotic value of $S h_{o}=64$ for all the mesh

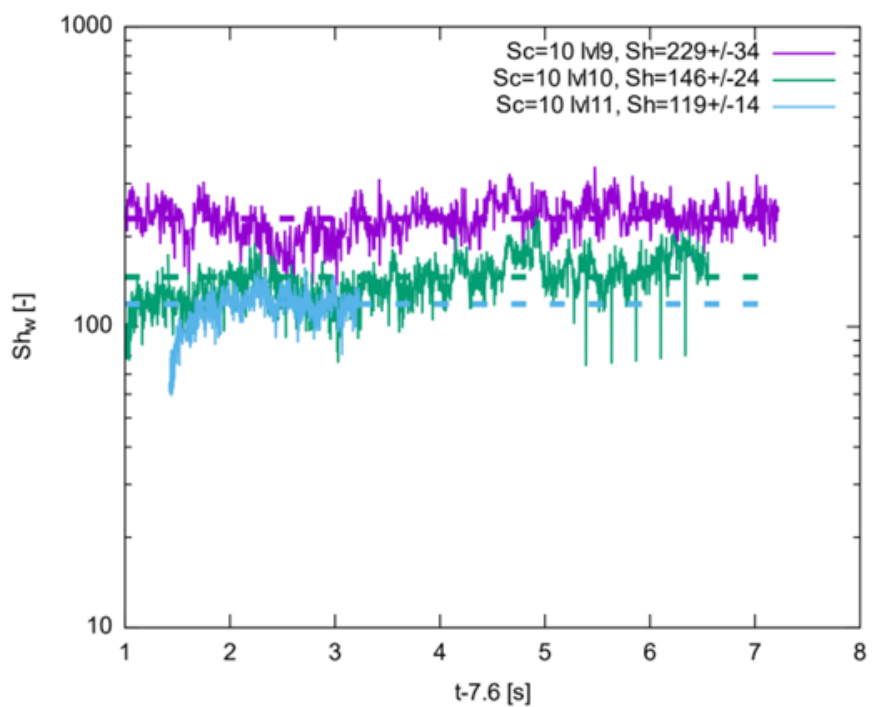

Fig. 14. Time evolution of the Sherwood number in the water phase $S h_{w, \text { num }}$ with different mesh resolutions at $S c_{w}=10$ and $Q=0.61 / \mathrm{min}$ and with $d_{i n j}=7.9 \mathrm{~mm}$.

resolutions. We can also note more fluctuations on the numerical Sherwood number in the oil phase that what could be observed in the water phase (cf. Fig. 14).

Using the time average numerical Sherwood number in the oil phase (cf. Sect. 8.3 and appendix 11.2 of [27]) and Table 4 we can compute (35) for each air flow rates. Then, we can plot the evolution of the ratio of the mass transfer coefficient as a function of the Froude number in Figure 18. We can see from Figure 18 that when the ratio of Schmidt number $S c_{r}=S c_{w} / S c_{o}$ decreases the ratio of time-averaged mass transfer coefficients $K_{w} / K_{o}$ increases but never goes higher than $K_{w} / K_{o}=3$. Considering $P=350$ corresponding to the partition coefficient of thymol between oil and water, and the water and oil volume of the experiment we can see that the approximation $\alpha \ll 1 / \beta$ remains valid in the Froude number range considered.

\subsubsection{Extrapolation of numerics to large Schmidt numbers}

First, we must keep in mind that to save computational time the numerical results are obtained for a maximum Schmidt number of $S c_{w}=40$. This means that we cannot directly compare our numerical results with the experimental one. Instead, we need to extrapolate our time average numerical Sherwood number to $S c_{w, t h}=1480$.

On Figure 19 we plot the numerically obtained Sherwood numbers for several Schmidt numbers at a constant air flow rate of $Q=0.6 \mathrm{l} / \mathrm{min}$. The error bars show the standard deviation of the numerical results from an asymptotic Sherwood number or time average Sherwood number $S h_{w}$ represented by dashed lines in Figures 14 and 15.

We can see that the three smallest Schmidt number results for the most resolved simulation $\left(\Delta_{\min }=131.75 \mu \mathrm{m}\right)$ align with a $S c^{5 / 12}$ law. This power law is remarkable as it 


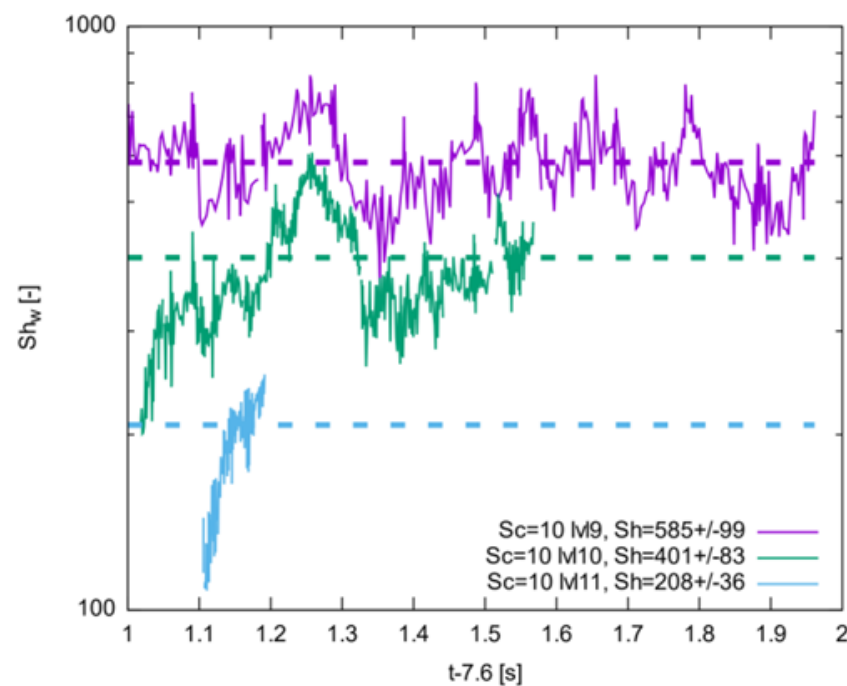

Fig. 15. Time evolution of the Sherwood number in the water phase $S h_{w, \text { num }}$ with different mesh resolutions at $S c_{w}=10$ and $Q=3.5 \mathrm{l} / \mathrm{min}$ and with $d_{i n j}=7.9 \mathrm{~mm}$.

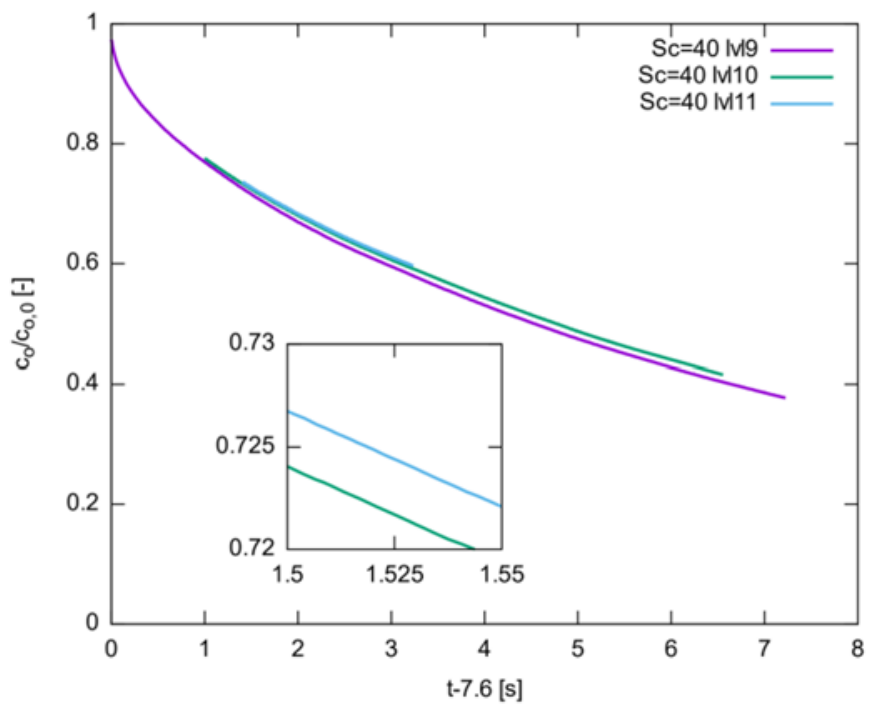

Fig. 16. Time evolution of the normalized concentration of tracer in the oil phase $C_{o} / C_{o, 0}$ with different mesh resolutions at $S c_{o}=40$ and $Q=0.6 \mathrm{l} / \mathrm{min}$ and with $d_{i n j}=7.9 \mathrm{~mm}$.

interpolates between the $S c^{1 / 2}$ law expected for mass transfer in a free surface flow and the $S c^{1 / 3}$ law expected for mass transfer in flow over a rigid (no slip) boundary. We expect the mass transfer behavior to interpolate between the $S c^{1 / 2}$ and the $S c^{1 / 3}$ law since the oil is much more viscous than water. Thus, relatively large chemical boundary layers in the water "see" a rigid oil phase while very thin boundary layers see a flowing oil phase. The interpolation would result in

$$
S h_{w}=a\left(S c^{1 / 2}+b S c^{1 / 3}\right)
$$

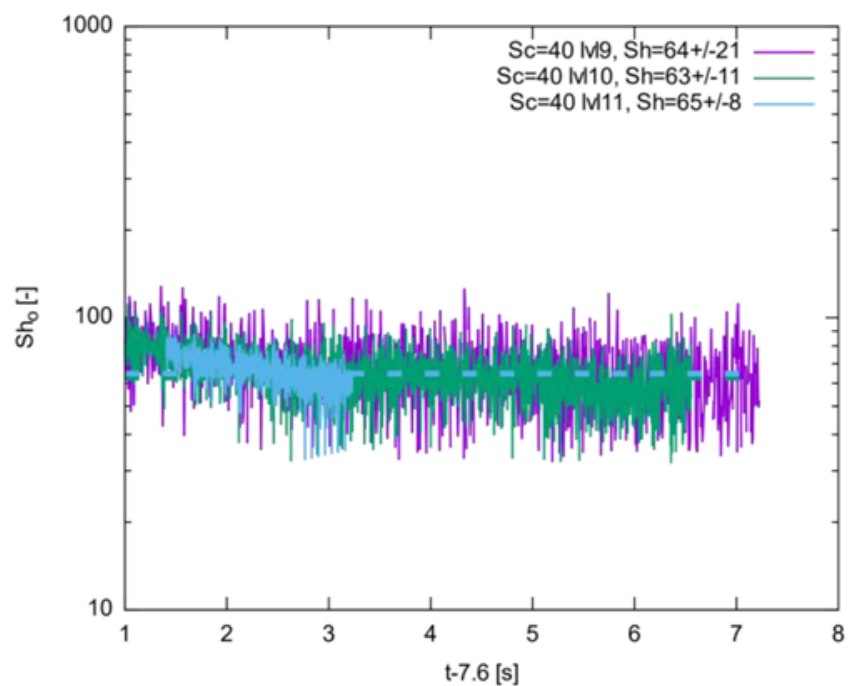

Fig. 17. Time evolution of the Sherwood number in the oil phase $S h_{o, \text { num }}$ with different mesh resolutions at $S c_{o}=40$ and $Q=0.6 \mathrm{l} /$ min and with $d_{i n j}=7.9 \mathrm{~mm}$.

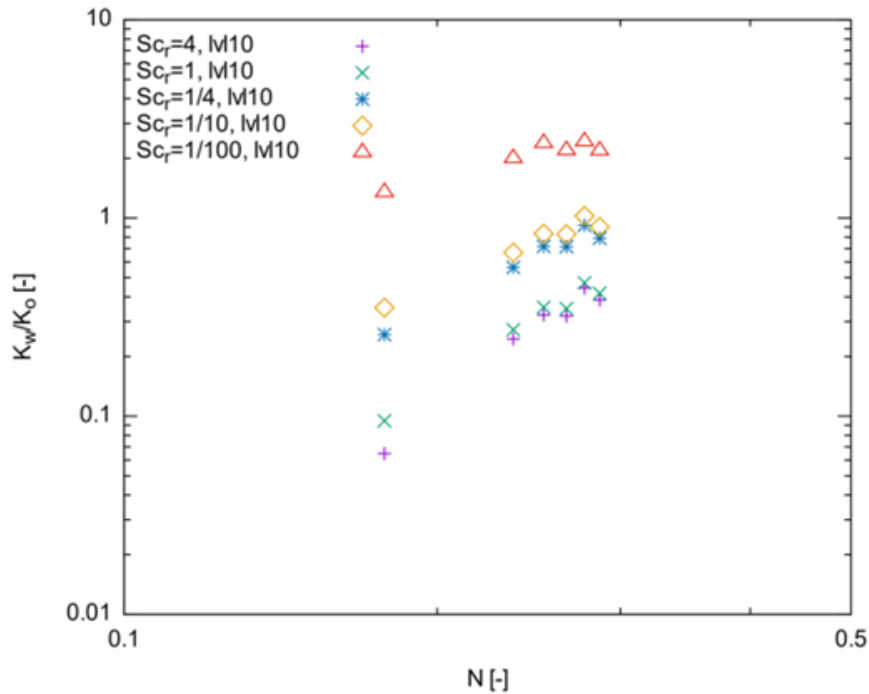

Fig. 18. Evolution of the ratio of time average mass transfer coefficient in the water and oil phase $\left(K_{w} / K_{o}\right)$ as a function of the Froude number for several ratios $S c_{r}=S c_{w} / S c_{o}$. The simulation results are obtained with a minimum grid size of $\Delta_{\min }=263.5 \mu \mathrm{m}$ corresponding to a maxlevel $=10$ of adaptive refinement.

where the coefficient $b$ depends on the ratio of viscosities of oil and water (or slag and metal) and the coefficient $a$ is obtained by fitting. The derivation of (36) will be published elsewhere and is still being perfected. However, it is likely that (36) can be approximated by a power law with an exponent $5 / 12$ that is the exact average of $1 / 3$ and $1 / 2$.

$$
S h_{w}=a S c^{5 / 12}
$$

where $a$ can be determined as the numerically obtained value for $S c=1$. This scaling gives extremely good results as shown on Figure 19, as it fits both the three first 
Table 5. Numerical values of the time averaged Sherwood number obtained at $S c=1$ and with a minimum grid size of $\Delta_{\min }=263.5 \mu \mathrm{m}$. Then, the value of $S h_{w, n u m}$ is extrapolated at $S c_{w, t h}=1480 \mathrm{using}$ (37) for various air flow rates.

\begin{tabular}{|c|c|c|c|c|}
\hline $\begin{array}{l}\text { Air flow rate } \\
Q(1 / \mathrm{min})\end{array}$ & $\begin{array}{l}\text { Froude number } \\
N\end{array}$ & $\begin{array}{l}\text { Sherwood number } \\
S h_{w, \text { num }} \text { at } S c=1\end{array}$ & Statistical error & $\begin{array}{l}\text { Sherwood number } \\
S h_{w, e x t} \text { at } S c w, t h=1480\end{array}$ \\
\hline 0.6 & 0.178 & 45 & 5 & 942 \\
\hline 2.5 & 0.237 & 59 & 8 & 1235 \\
\hline 3.5 & 0.253 & 74 & 11 & 1549 \\
\hline 4.5 & 0.266 & 77 & 10 & 1612 \\
\hline 5.5 & 0.277 & 100 & 14 & 2094 \\
\hline 6.5 & 0.277 & 107 & 11 & 2240 \\
\hline
\end{tabular}

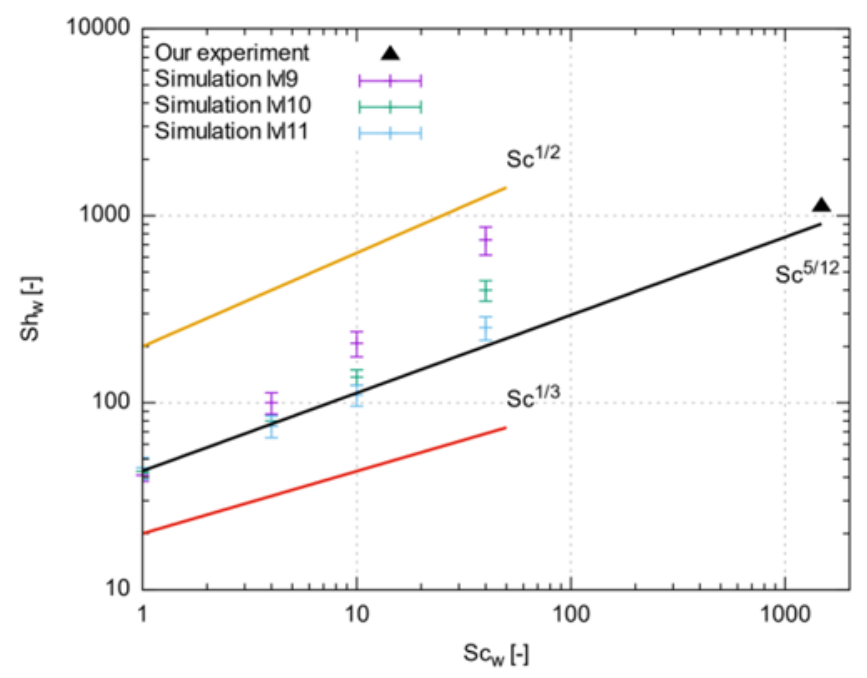

Fig. 19. Dependency of the time averaged numerical Sherwood number on the Schmidt number for different mesh refinements and $Q=0.6 \mathrm{l} / \mathrm{min}$ and with $d_{i n j}=7.9 \mathrm{~mm}$.

numerical data points for $S c=1,4$ and 10 and the experimental one at $S c_{w, t h}=1480$. The values of the Sherwood numbers for various air flow rates extrapolated in this manner for $S c_{w, t h}=1480$ are gathered in Table 5 .

However, these good results could be the effect of two compensating errors: the spatial discretization error and the extrapolation error. A "safer" manner of extrapolating the low Sc results is to consider the two limiting scaling laws as upper and lower bounds so that

$$
a S c^{1 / 3}<S h_{w}<a S c^{1 / 2}
$$

where $a$ is the value for $S c=1$. Therefore, we plot the extrapolation of the numerical results with the two limiting scaling laws as error bars on Figure 20.

From the limited number of numerical results that we have, we cannot obviously observe both mass transfer regimes seen experimentally (cf. Fig. 20) but only the first regime. However, we notice a steep increase of the Sherwood numbers for the two largest air flow rates, indicative of the beginning of a transition to the second regime.

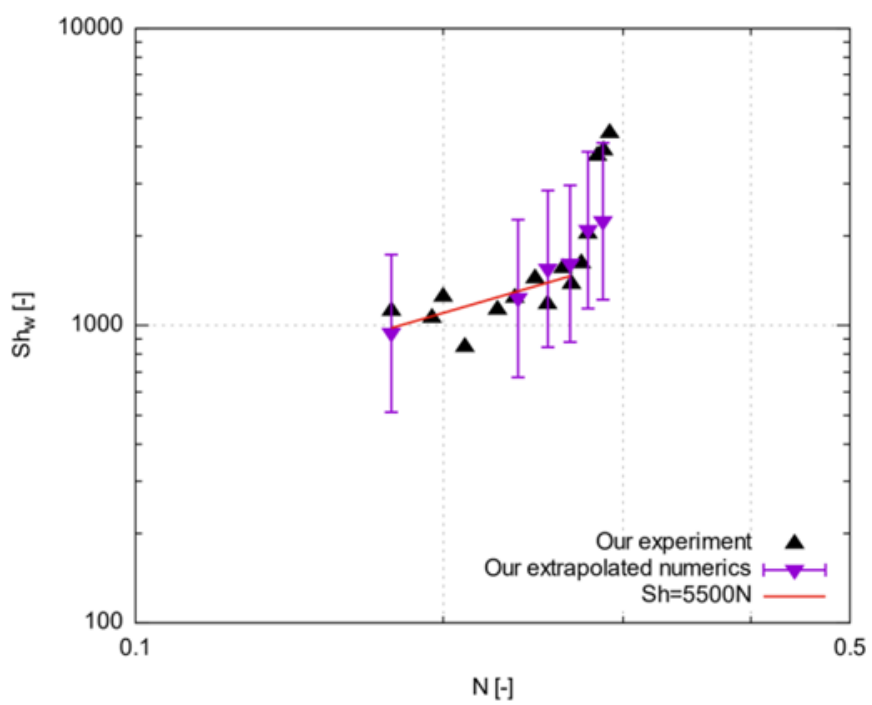

Fig. 20. Time averaged experimental Sherwood number (black triangles) and extrapolation at $S c_{w, t h}=1480$ of the time averaged numerical Sherwood number values (purple triangles with purple error bars) as a function of the modified Froude number.

Finally, we want to verify the assumption made on the Section 3.2.1 concerning the role of the water-oil interfacial area in the mass transfer regime change. As we can see from the numerical results of Figure 21 when the air flow rate increases the water-oil interfacial area decreases. However, above a critical air flow rate $Q>5.5 \mathrm{l} / \mathrm{min}$, we observe that the interfacial area reaches a value close to the interfacial area obtained at the lowest flow rate. Now, if we normalize the water-oil interfacial area with the ratio of the difference of the area of the section of the box with the time average open eye area in Figure 22. We can see that when the air flow rate is increased the normalized interfacial area increases. This is more striking for $Q \geq 5.5 \mathrm{l} / \mathrm{min}$ where we can see a steep increase. On the other hand, the values of the normalized interfacial area at low air flow rates are close to one. This indicates that despite having a bigger open eye in the oil layer, the numerous oil droplets in the water increase well the normalized interfacial area at high air flow rate compared to low flow rate. So, from the 


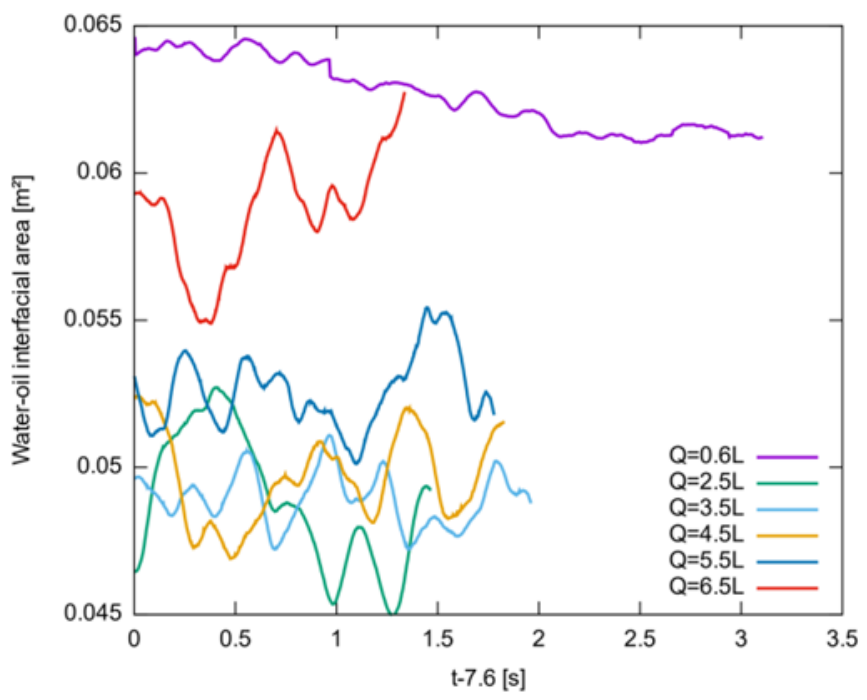

Fig. 21. Time evolution of the numerical water-oil interfacial area for different air flow rates. The results are obtained with a constant minimum grid size of $\Delta_{\min }=527 \mu \mathrm{m}$ corresponding to a maxlevel $=9$ of adaptive refinement.

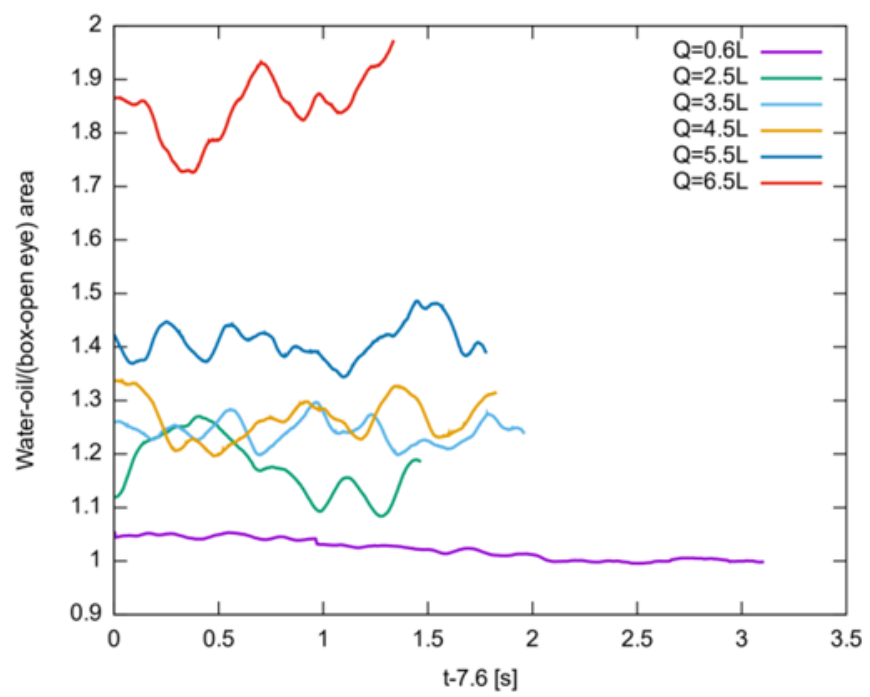

Fig. 22. Time evolution of the ratio of the numerical water-oil interfacial area to the difference of the area of the section of the box with the time average open eye area for different air flow rates. The results are obtained with a constant minimum grid size of $\Delta_{\min }=527 \mu \mathrm{m}$.

numerical results the change in the normalized interfacial area plays an important role in the increase of the Sherwood number.

\section{Conclusion}

In this study, we have investigated the mass transfer between water and oil as a model of the one between liquid steel and slag in an industrial process. To reproduce this complex industrial process, we designed an experiment at ambient temperature using water and oil instead of metal and slag. We used thymol in solution as a chemical tracer transferred from the water to the oil layer. We have also designed a Direct Numerical Simulation of the experimental configuration in addition to this experiment. We have first verified that we could reproduce the hydrodynamic behavior of the industrial process by measuring the evolution of the open eye area as a function of the air flow rate. Comparison with the literature reveals that one observes areas close to those observed in other water experiments. The literature also shows that the open eye area is very strongly affected by parameters such as the fluid density. Moreover, our numerical results for the open eye area give an approximate agreement with the experimental ones, showing the same logarithmic trend and an error limited to $20 \%$.

In the second part of this paper, we measured the mass transfer of thymol from the water to the oil phase using the same experimental configuration. Our experimental results show that two different mass transfer regimes can be identified depending on the air flow rate value. Furthermore, the transition between the two regimes coincides with the critical Froude number for the entrainment of oil droplets into the water. This agrees with the results reported in the literature.

Finally, the mass transfer of the water experiment was simulated. We used an extrapolation of our numerical results at a Schmidt number $S c=1$ to the experimental Schmidt number $S c_{w, t h}=1480$. This led to some encouraging results, in agreement with the experiments although with large error bars. However, from the limited numerical results we obtained we could not clearly observe the second mass transfer regime. Nevertheless, at high air flow rate the results show something similar to a transition region between the two regimes.

For the first mass transfer regime the numerics and the experiments are well predicted by the following correlation

$$
S h_{w}=5500 N .
$$

This linear law (exponent of unity) should be compared with the correlations found by our analysis of the literature in Figure 11, which involves exponents 1.8, 1.9 and 3.1. A definite disagreement between the various authors is noticed while our experiment (exponent 0.8) agrees with our numerics. The causes of this intriguing disagreement should be the object of future study, including numerical simulation of the other author's experiments.

\section{Nomenclature}

\section{Greek symbols}

$\rho \quad$ Density, $\left[\mathrm{kg} / \mathrm{m}^{3}\right]$.

$\mu$ Dynamic viscosity, $[\mathrm{kg} / \mathrm{m} . \mathrm{s}]$.

$v$ Kinematic viscosity, $\left[\mathrm{m}^{2} / \mathrm{s}\right]$.

$\sigma$ Surface tension, $[\mathrm{N} / \mathrm{m}]$.

$\chi$ Volume fraction, $[-]$.

$\kappa$ Curvature, $\left[\mathrm{m}^{-1}\right]$.

$\delta_{s}$ Dirac distribution function. 
$\varepsilon \quad$ Rate of dissipation of turbulent kinetic energy, $\left[\mathrm{m}^{2} / \mathrm{s}^{3}\right]$.

$\eta \quad$ Kolmogorov length scale, $[\mathrm{m}]$.

$\Delta$ Mesh size, $[\mathrm{m}]$.

Latin symbols

$n \quad$ Normal to the interface, $\left[\mathrm{m}^{-1}\right]$.

$L \quad$ Width of the ladle, [m].

$h$ Height of fluid, [m].

$d \quad$ Diameter, [m].

$V \quad$ Volume of fluid, $\left[\mathrm{m}^{3}\right]$.

$S \quad$ Spreading parameter $[\mathrm{N} / \mathrm{m}]$.

$Q$ Gas flow rate at operating condition and injector height, $\left[\mathrm{m}^{3} / \mathrm{s}\right]$.

$g \quad$ Gravitational acceleration, $\left[\mathrm{m} / \mathrm{s}^{2}\right]$.

$J \quad$ Mass flux, $\left[\mathrm{kg} / \mathrm{m}^{2} \mathrm{~s}\right]$.

$A$ Interfacial area, $\left[\mathrm{m}^{2}\right]$.

$C$ Concentration of tracer, [g/l].

$D$ Diffusion coefficient, $\left[\mathrm{m}^{2} / \mathrm{s}\right]$.

$K \quad$ Global mass transfer coefficient, $[\mathrm{m} / \mathrm{s}]$.

$U_{p} \quad$ Velocity of bubble plume, $[\mathrm{m} / \mathrm{s}]$.

$u$ Velocity, $[\mathrm{m} / \mathrm{s}]$.

$T$ Numerical tracer concentration, [g/l].

$P \quad$ Partition coefficient, $[-]$.

Fr Froude number, [-].

$N$ Re-expression of the Froude number [-].

Sc Schmidt number, [-].

Sh Sherwood number, [-].

$m$ Viscosity ratio, [-].

$r \quad$ Density ratio, $[-]$.

$S c_{r}$ Schmidt ratio, [-].

\section{Sub/superscripts}

$x \quad$ Horizontal dimension.

a Air.

$w$ Water.

$o$ Oil.

a Air.

$m$ Steel or equivalent phase.

$s \quad$ Slag or equivalent phase.

c Concentration.

0 Initial condition.

th Thymol.

, Interface value.

inj Injector.

il Industrial ladle.

$r l$ Reduced size ladle.

Time average value.

$\infty \quad$ Fluid bulk.

Acknowledgments. This work was supported by ANRT with convention CIFRE $N^{\circ} 2017 / 0694$ and benefited from access to the HPC resources of CINES under the allocations 2018A0052B07760 and 2019 - A0072B07760 and the resources of the TGCC under the project 2020225418 granted respectively by GENCI and PRACE. Support by the ERC ADV grant TRUFLOW is acknowledged.

\section{Supplementary Material}

Figures 1-23.

The Supplementary Material is available at https://www.metal. org $/ 10.1051 /$ metal $/ 2021088 /$ olm.

\section{References}

1. P. Riboud, R. Vasse, Désulfuration de l'acier en poche: synthèse des résultats théoriques et industriels, Revue de Métallurgie. 82, 801-810 (1985)

2. S.-H. Kim, R.J. Fruehan, Physical modeling of liquid/liquid mass transfer in gas stirred ladles, Metall. Trans. B 18, 381390 (1987)

3. J. Ishida, Effects of stirring by argon gas injection on metallurgical reactions in secondary steelmaking, DenkiSeiko (Electr. Furn. Steel) 52, 2-8 (1981)

4. J. Mietz, S. Schneider, F. Oeters, Model experiments on mass transfer in ladle metallurgy, Steel Res. 62, 1-9 (1991)

5. M. Hirasawa, K. Mori, M. Sano, A. Hatanaka, Y. Shimatani, Y. Okazaki, Rate of mass transfer between molten slag and metal under gas injection stirring, Trans. Iron Steel Inst. Jpn, 27, 277-282 (1987)

6. H. Lachmund, Y. Xie, T. Buhles, W. Pluschkell, Slag emulsification during liquid steel desulphurisation by gas injection into the ladle, Steel Res. Int. 74, 77-85 (2003)

7. D. Bothe, M. Koebe, K. Wielage, J. Prüss, H.-J. Warnecke, Direct numerical simulation of mass transfer between rising gas bubbles and water, in Bubbly Flows (Springer, 2004), pp. $159-174$

8. W. Lou, M. Zhu, Numerical simulation of desulfurization behavior in gas-stirred systems based on computation fluid dynamics-simultaneous reaction model (CFD-SRM) coupled model, Metall. Mater. Trans. B 45, 1706-1722 (2014)

9. W. Lou, M. Zhu, Numerical simulation of slag-metal reactions and desulfurization efficiency in gas-stirred ladles with different thermodynamics and kinetics, ISIJ Int. 55, 961-969 (2015)

10. R.V. Calabrese, T. Chang, P. Dang, Drop breakup in turbulent stirred-tank contactors. Part I: Effect of dispersedphase viscosity, AIChE J. 32, 657-666 (1986)

11. R. Calabrese, C. Wang, N. Bryner, Drop breakup in turbulent stirred-tank contactors. Part III: Correlations for mean size and drop size distribution, AIChE J. 32, 677-681 (1986)

12. C. Wang, R.V. Calabrese, Drop breakup in turbulent stirredtank contactors. Part II: Relative influence of viscosity and interfacial tension, AIChE J. 32, 667-676 (1986)

13. M. Iguchi, Y. Sumida, R. Okada, Z. Morita, Evaluation of critical gas flow rate for the entrapment of slag using a water model, ISIJ Int. 34, 164-170 (1994)

14. A. Monin, A.M. Yaglom, Statistical fluid mechanics (MIT Press, Cambridge, MA, 1971), vols. 1 and 2, p. 11

15. G.R. Hunt, T.S. van den Bremer, Classical plume theory: 1937-2010 and beyond, IMA J. Appl. Math. 76, 424-448 (2010)

16. S. Popinet, Gerris: a tree-based adaptive solver for the incompressible Euler equations in complex geometries, J. Comput. Phys. 190, 572-600 (2003) 
17. S. Popinet, An accurate adaptive solver for surface-tensiondriven interfacial flows, J. Comput. Phys. 228, 5838-5866 (2009)

18. J.U. Brackbill, D.B. Kothe, C. Zemach, A continuum method for modeling surface tension, J. Comput. Phys. 100, 335-354 (1992)

19. D.C. Kurt, A. Smith, F.J. Solis, A projection method for motion of triple junctions by level sets, Interfaces Free Boundaries (2002)

20. X. Chen, Y. Sun, C. Xue, Y. Yu, G. Hu, Tunable structures of compound droplets formed by collision of immiscible microdroplets, Microfluid. Nanofluid. 21, 109 (2017)

21. J. López-Herrera, A. Gañán-Calvo, S. Popinet, M. Herrada, Electrokinetic effects in the breakup of electrified jets: a volume-of-fluid numerical study, Int. J. Multiphase Flow 71, 14-22 (2015)

22. S.B. Pope, Turbulent Flows (IOP Publishing, 2001)
23. K. Yonezawa, K. Schwerdtfeger, Spout eyes formed by an emerging gas plume at the surface of a slag-covered metal melt, Metall. Mater. Trans. B 30, 411-418 (1999)

24. K. Krishnapisharody, G.A. Irons, Modeling of slag eye formation over a metal bath due to gas bubbling, Metall. Mater. Trans. B 37, 763-772 (2006)

25. M. Thunman, S. Eckert, O. Hennig, J. Björkvall, D. Sichen, Study on the formation of open-eye and slag entrainment in gas stirred ladle, Steel Res. Int. 78, 849-856 (2007)

26. D. Bonn, J. Eggers, J. Indekeu, J. Meunier, E. Rolley, Wetting and spreading, Rev. Mod. Phys. 81, 739 (2009)

27. N. Joubert, Liquid-liquid mass transfer characterization applied to metallurgical process, $\mathrm{PhD}$ dissertation, Sorbonne Université/Université Pierre et Marie Curie Paris VI (2021). https://hal.archives-ouvertes.fr/tel03227840

Cite this article as: Nelson Joubert, Pascal Gardin, Stéphane Popinet, Stéphane Zaleski, Experimental and numerical modelling of mass transfer in a refining ladle, Metall. Res. Technol. 119, 109 (2022) 